\title{
Article
}

\section{Large Molecular Cluster Formation from Liquid Materials and Its Application to ToF-SIMS}

\author{
Kousuke Moritani * (D), Shogo Nagata, Atsushi Tanaka, Kosuke Goto and Norio Inui \\ Graduate School of Engineering, University of Hyogo, 2167 Shosha, Himeji, Hyogo 671-2201, Japan; \\ es14e030@steng.u-hyogo.ac.jp (S.N.); ej17t013@steng.u-hyogo.ac.jp (A.T.); goto@eng.u-hyogo.ac.jp (K.G.); \\ inui@eng.u-hyogo.ac.jp (N.I.) \\ * Correspondence: moritani@eng.u-hyogo.ac.jp; Tel.: +81-79-267-4921
}

check for updates

Citation: Moritani, K.; Nagata, S.; Tanaka, A.; Goto, K.; Inui, N. Large Molecular Cluster Formation from Liquid Materials and Its Application to ToF-SIMS. Quantum Beam Sci. 2021, 5, 10. https://doi.org/10.3390/ qubs5020010

Academic Editor: Klaus-Dieter Liss

Received: 12 March 2021

Accepted: 27 April 2021

Published: 29 April 2021

Publisher's Note: MDPI stays neutral with regard to jurisdictional claims in published maps and institutional affiliations.

\begin{abstract}
Since molecular cluster ion beams are expected to have various chemical effects, they are promising candidates for improving the secondary ion yield of Tof-SIMS. However, in order to clarify the effect and its mechanism, it is necessary to generate molecular cluster ion beams with various chemical properties and systematically examine it. In this study, we have established a method to stably form various molecular cluster ion beams from relatively small amounts of liquid materials for a long time by the bubbling method. Furthermore, we applied the cluster ion beams of water, methanol, methane, and benzene to the primary beam of SIMS and compared the molecular ion yields of aspartic acid. The effect of enhancing the yields of $[\mathrm{M}+\mathrm{H}]^{+}$ion of aspartic acid was found to be the largest for the water cluster and small for the methane and benzene clusters. These results indicate that the chemical effect contributes to the desorption/ionization process of organic molecules by the molecular cluster ion beam.
\end{abstract}

Keywords: SIMS; cluster ion beam; desorption/ionization; water cluster; molecular cluster; mixed cluster

\section{Introduction}

ToF-SIMS has become a unique surface analysis method that can detect high-mass molecular ions since the large cluster ions such as argon (Ar) cluster ion beam [1] was commercialized as the primary ion of SIMS. The advantages of large cluster ion beams are less damage to the organic sample during sputtering [2] and the ability to detect higher mass secondary ions [3]. Now it is expected as a powerful tool for chemical imaging of biological samples and organic materials in medical and biological fields. However, in order to detect and image the distribution of small amounts of biopolymers in a sample with high sensitivity and high resolution, it is necessary to further increase the yield of secondary ions. Therefore, improving the secondary ion yield is still an important issue for SIMS.

For the improvement of the secondary ion yield, some researchers have tried to use molecular clusters [4-7] instead of inert Ar cluster or clusters mixed with highly reactive molecules [8,9] in expectation of chemical effects. Among these challenges, a water cluster [5-7] is the most successful case. It has been reported that the molecular ion yields enhanced by several tens to 1000 fold or more for some molecules. However, in order to apply the chemical imaging using SIMS to the fields of medical science and biology, further increase of the molecular ion yield is required. Furthermore, the mechanism of the chemical effects for desorption and ionization of organic molecules by the primary ion beams is not well understood, and then no effective method for improving the ionization rate has been established yet. The reaction dynamics at the moment of cluster collision is difficult to observe directly, because there are multi-body non-equilibrium processes that occur in a very short time. However, systematically examining the increase or decrease in 
secondary ion yield by colliding various molecular clusters which have different chemical properties will provide important information for elucidating the ionization mechanism.

In this study, we have established a method to stably form various molecular cluster ion beams for a long time from a relatively small amount of liquid material by adopting the bubbling method. This paper describes the formation of the cluster ion beams of water, methanol, and benzene. In addition, we applied these cluster ion beams to the primary beam of ToF-SIMS and compared the molecular ion yields of aspartic acid.

The chemical effect of the beam will depend on the combination of primary ions and target molecules. However, the dynamics of desorption/ionization of organic molecules by the collision of molecular clusters is still not clear, and thus it is difficult to predict their effects. The techniques for the stable formation of various types of molecular cluster ion beams enable the systematic study of the chemical effects of primary ion beams, opening the door for exploring new SIMS projectiles.

\section{Experimental}

Details of the Ar cluster ion beam and ToF-SIMS apparatus are given elsewhere [4,10]. Briefly, the apparatus consisted of three parts: a cluster-source chamber, an ionization and cluster-size-selection chamber, and a sample chamber. All parts were differentially pumped. The base pressure of the sample chamber was $8 \times 10^{-8} \mathrm{~Pa}$. Before measuring mass spectra of ions detected under surface bombardment with cluster ions at the sample chamber, the target surfaces were etched sufficiently with the Ar cluster ion beam to remove surface contaminants.

A brass conical nozzle with a $0.1 \mathrm{~mm}$ diameter aperture and $60 \mathrm{~mm}$ expansion and cooling zone was attached in the source chamber. The neutral cluster is generated by adiabatic expansion from the nozzle. The clusters were ionized by electron impact from a tungsten filament at an energy of $150 \mathrm{eV}$ and then, extracted at an acceleration voltage of $5 \mathrm{kV}$. We selected the required cluster size $(n)$ by employing a time-of-flight (ToF) technique using two ion deflectors. Here, the cluster size indicates the number of cluster constituent particles. The cluster size resolution $(n / \Delta n)$ of more than ten was achieved by shortening the ion deflectors' pulse width. The methods of the Ar [10] and methane and $\mathrm{Kr}$ [4] cluster beam generation is described in our previous paper. Briefly, methane gas $(10 \%$ concentration) was seeded in Ar gas (90\% concentration) for cooling, and the mixed gas is ejected from the nozzle.

In this study, cluster ion beams of water, methanol, and benzene were generated and used as a primary ion beam of ToF-SIMS. These molecules do not expand adiabatically from the nozzle due to the low vapor pressure usually. The techniques for forming clusters from liquid materials in a vacuum could be divided into three major types: bubbling, baking, and electrospray method. The baking method requires a relatively large volume of the liquid heating reservoir. The electrospray method requires a different nozzle setup from the Ar cluster formation, such as capillaries. Thus, we adopted the bubbling method for generating the water, methanol, and benzene clusters in this study. The $100 \mathrm{~mL}$ of liquids (water (distilled water, Wako) and $\mathrm{D}_{2} \mathrm{O}$ (purity: 99.9\%, Wako), methanol (purity: 99.8\%, Wako), and benzene (purity: 99.5\%, Wako)) were sealed in a stainless steel reservoir in the gas line and kept at room temperature. The liquid was bubbled and vaporized by the Ar carrier gas, and the gas mixture was adiabatically expanded from the nozzle to form clusters. The flow rate of the Ar carrier gas was controlled by a mass flow controller. The chemical compositions of the beams were estimated from the change in the component of the background gas in the sample chamber when the beam was on and off by the deflector. Since the sensitivity of QMS varies depending on the molecule due to the ionization rate, we have estimated the Ar mixing ratio per charge $\left(C_{\mathrm{Ar}}\right)$ of the cluster ion beams from the equation:

$$
C_{\mathrm{Ar}}=\left(\Delta P_{\mathrm{Ar}-\mathrm{mix}} / I_{\text {mix }}\right) /\left(\Delta P_{\mathrm{Ar}-\mathrm{Ar}} / I_{\mathrm{Ar}}\right) \times 100 .
$$

Here, $I_{\text {mix }}$ and $I_{\mathrm{Ar}}$ are the ion beam currents of the cluster ion beam generated from the mixed gas and generated from the pure Ar gas. The ion beam currents were measured 
using a Faraday cup placed at the sample position. $\Delta P_{\mathrm{Ar}-\mathrm{mix}}$ and $\Delta P_{\mathrm{Ar}-\mathrm{Ar}}$ are the Ar partial pressure changes when each beam enters into the sample chamber, which were measured by the quadrupole mass spectrometer (QMS) equipped with the sample chamber.

As a target sample for SIMS measurements, thin films of aspartic acid (Asp; $\mathrm{C}_{4} \mathrm{H}_{7} \mathrm{NO}_{4}$, molecular weight; 133.10) were used. The Asp film was prepared by dropping $20 \mu \mathrm{L}$ of an Asp aqueous solution $(1 \mathrm{~g} / \mathrm{L})$ onto a Si substrate and freeze-drying. The film thickness measured with a stylus-type profiler (DekTak-3, Veeco) was 1-4 $\mu \mathrm{m}$. Samples were not prepared under controlled conditions such as in a clean room, so it is necessary to consider the contamination of the laboratory air and container materials. In this experiment, typical contaminants such as $\mathrm{Na}$ and polydimethylsiloxane (PDMS) were detected by SIMS. Therefore, in order to remove these contaminants, the experiments were performed after irradiating Ar cluster ions with $1.0 \times 10^{13}$ ions $/ \mathrm{cm}^{2}$ at an accelerating voltage of $5 \mathrm{kV}$ and sputtering the sample surface before SIMS measurement. Consequently, almost all the contaminants by PDMS have been removed by cluster sputtering, but it was difficult to remove Na contamination.

\section{Results and Discussion}

Figure 1 shows the background intensity of the mass peaks at $m / z$ of 18 and 40 in the sample chamber when the Ar cluster ion beam entered (on) and was shut off (off) by the deflector. The flow rate of Ar gas was $260 \mathrm{sccm}$. Only the partial pressure of $\operatorname{Ar}(\mathrm{m} / \mathrm{z}=40)$ changes with the beam on and off.

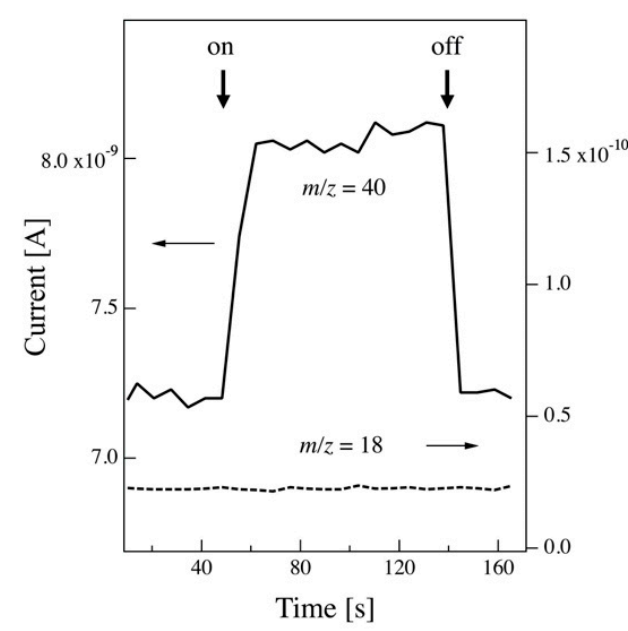

Figure 1. The background intensity of the $m / z=18$ and 40 mass peaks in the sample chamber when the Ar cluster ion beams enter (on) and are shut off (off) by the deflector.

Figure 2 shows when the water cluster ion beams are on and off. Ar flow rates are 180 (a) and 280 (b) sccm. In Figure $2 \mathrm{a}, \mathrm{H}_{2} \mathrm{O}$ increases and decreases when the beam is turned on and off, but Ar does not change, which indicates that pure water clusters were generated despite using mixed gas. On the other hand, in Figure $2 b$, both $\mathrm{H}_{2} \mathrm{O}$ and $\mathrm{Ar}$ increase and decrease simultaneously with the beam on and off. This indicates that water and Ar are included in the cluster ion beam at a higher Ar flow rate. The Ar content of each cluster was calculated according to Equation (1). The Ar content was $0 \%$ when the Ar flow rate was below $180 \mathrm{sccm}$. As the Ar flow rate increased further above $190 \mathrm{sccm}$, the Ar content increased, reaching $76 \%$ at $280 \mathrm{sccm}$. We performed the same experiment with methanol and calculated the Ar content in the methanol cluster ion beams.

Figure 3 shows the Ar and Ne flow rate dependence of the Ar content in the cluster beam (a) and ion beam current (b) for water and methanol cluster generated using Ar or $\mathrm{Ne}$ carrier gas. In the case of the water cluster, pure water cluster beams are generated below the Ar flow rate of $180 \mathrm{sccm}$, and mixed cluster beams of water and Ar are generated above $190 \mathrm{sccm}$. In the case of methanol, pure methanol cluster beams are formed below 
$220 \mathrm{sccm}$. As shown in these figures, the content of Ar in the cluster beams increases with increasing the Ar flow rate. The beam current decreases once Ar begins to mix into the cluster and then increases again.

Although the adiabatic expansion of gas flow should generate a cluster, a theory to predict cluster formation in gas jets has yet to be established. However, an empirical scaling parameter $\Gamma^{*}$ can describe the condition to produce a cluster $[11,12]$. The average cluster size $<n>$ is:

$$
<n>=33\left\{\Gamma^{*} / 1000\right\}^{2.35} \text {. }
$$

$\Gamma^{*}$ is proportional to the so-called "Hagena parameter" $k$, which is a constant related to bond formation. Hence, $\Gamma^{*}$ depends on the gas species. The water and the methanol molecules form hydrogen bonds which are stronger than van der Waals forces and then form clusters more easily than Ar. Therefore, when the mixture of these molecules and Ar gas expands adiabatically, the water or the methanol clusters grow before the growth of Ar clusters. It is known that in a two-component supersonic beam, the heavy component increases significantly on the beam center line, and the light component is ejected to the fringe [13]. In our apparatus, the skimmer is $25-\mathrm{mm}$ downstream from the nozzle, and cuts off only a small portion around the beam center from the total nozzle flow. As a result, the concentration of the pure water cluster may considerably increase in the sample chamber. As the Ar flow rate increases, the number of Ar atoms which collide during expansion at the nozzle aperture increases, and thus, the probability of Ar clustering should increase. Therefore, a mixed cluster composed of Ar atoms and the other molecules is generated at a high Ar flow rate. The formation of a mixed cluster can be concluded from the dissociative scattering mass spectrum of the cluster ions, which will be explained later.

The relationship between the Ar concentration per charge and the beam current of the water/Ar mixed cluster can be interpreted as follows. When Ar atoms begin to mix in a cluster, small amounts of Ar disturb the formation of stable molecular cluster phase and then the beam current decreases. As the Ar content in a cluster increases, each layer of the molecules and argon stabilizes, and clusters should easily form. Then, the beam current increases again. It has been reported that a mixed cluster of water and Ar has a structure of Ar coated water clusters [14]. However, since $k$ is a parameter relating to bond formation and depends on the chemical species, the flow rate dependency of the mixing ratio differs depending on the ratio of the cohesive energy of molecules to carrier gas.

In the case of $\mathrm{Ne}$, the water cluster did not contain $\mathrm{Ne}$ below the $\mathrm{Ne}$ flow rate of $320 \mathrm{sccm}$. This is because the Ne is less likely to aggregate than Ar. At the flow rate of $300 \mathrm{sccm}$, almost the same ion beam current was obtained as when the Ar flow rate was $180 \mathrm{sccm}$ [15]. We also performed the same experiments using helium $(\mathrm{He})$ as carrier gases. However, no clusters were obtained under the same range of the flow rate. When the gas molecules are emitted from a small enough aperture compared to its mean free path, multiple collisions of the carrier gas occur. Since the mass of He is close to hydrogen, He can easily excite $\mathrm{H}-\mathrm{O}$ vibrations in the event of a collision, and as a result, it may not reduce the internal energy of water molecules (see Appendix A).

In order to generate a higher-purity water cluster, it should be better to use $\mathrm{Ne}$ as a carrier gas. However, the advantage of using Ar gas is that Ar is inexpensive and the mixing ratio can be adjusted by changing the flow rate. Therefore, Ar was used as carrier gas in this study. The advantage of the bubbling method is that a cluster beam can be generated stably for a long time with a small amount of liquid material. We have confirmed that $100 \mathrm{~mL}$ of distilled water can stably generate the water cluster ion beam totally for far more than several tens hours at the Ar flow rate of $180 \mathrm{sccm}$. 

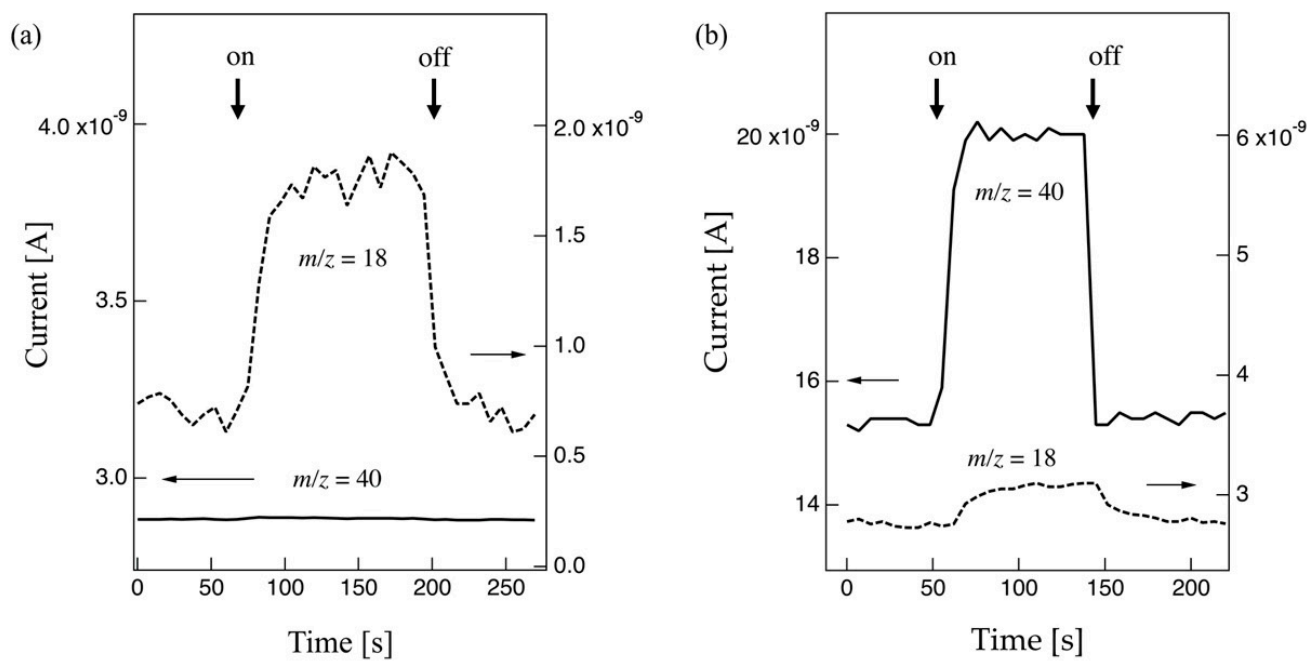

Figure 2. The background intensity of the $m / z=18$ and 40 mass peaks in the sample chamber when the water cluster ion beams enter (on) and are shut off (off) by the deflector. Ar flow rates are 180 (a) and 280 (b) sccm. Data in (a) are taken from Ref. [15].

(a)

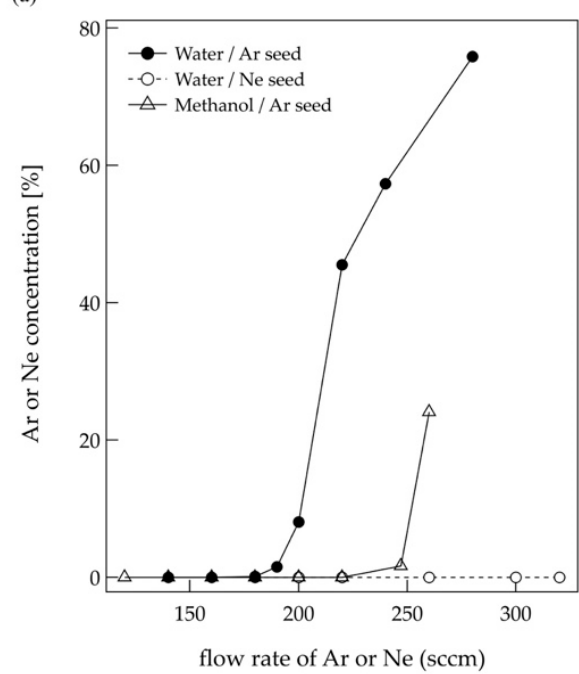

(b)

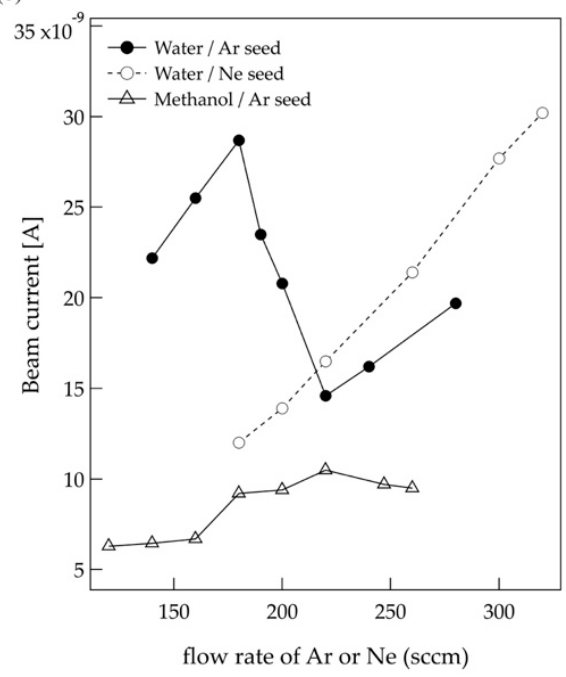

Figure 3. Ar/Ne flow rate dependence of $\mathrm{Ar} / \mathrm{Ne}$ concentration per charge (a) and the beam current (b) for the water and the methanol cluster ion beams. Data for water clusters are taken from Ref. [15].

Figure 4 shows the typical size distribution of water, methanol, and benzene clusters generated at an Ar flow rate of $180 \mathrm{sccm}$. The distribution curves are normalized at the peak top. The $\mathrm{n}$ means the number of constituent molecules of a cluster. The distribution of $\mathrm{n}$ was obtained by converting the $\mathrm{m} / \mathrm{z}$ distribution measured using the ToF method. As shown in this figure, $n=570-2350$ for water, 990-3450 for methanol, and 1100-3900 for benzene with a full width at half maximum (FWHM), respectively. The cluster size can be adjusted by changing the temperature of the liquid reservoir and gas line. Heating above $388 \mathrm{~K}$ increased cluster size and beam current. On the other hand, the liquid material was consumed in a short time. In this study, the gas line was kept at room temperature, and clusters were stably formed for an extended period. 


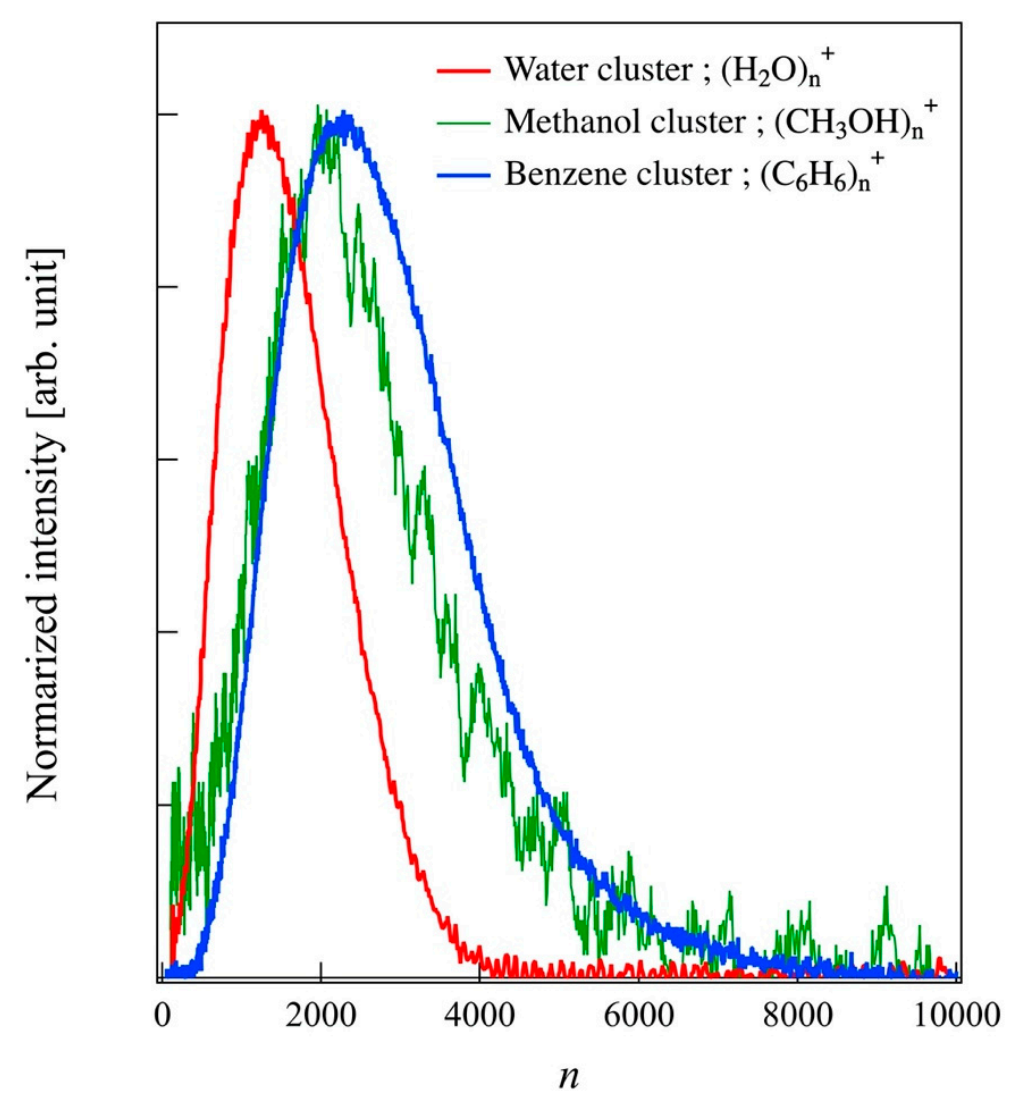

Figure 4. Size (n) distribution of water, methanol, and benzene cluster ion beams at an Ar flow rate of $180 \mathrm{sccm}$ before size selection.

Figure 5 shows the mass spectra of dissociative cluster ions scattered from a silver (Ag) sample under bombardment with the size-selected water $\left(\mathrm{D}_{2} \mathrm{O}\right)$ cluster ions at an incident angle of $45^{\circ}$ at the $n=900$ (energy per molecule; $E / n=5.6 \mathrm{eV}$ ). A series of peaks associated with the decomposed water cluster ions can be observed in the mass spectra. A periodic series of the mass peaks $\left[\left(\mathrm{D}_{2} \mathrm{O}\right)_{\mathrm{n}}+\mathrm{D}\right]^{+}$indicates that the clusters decompose during the surface collisions, however, the molecules themselves do not dissociate. At higher $E / n$, the clusters were broken down into smaller clusters, but the molecules themselves did not dissociate even at the $E / n=10.0 \mathrm{eV}$. The detected multimer ions of $\mathrm{D}_{2} \mathrm{O}$ molecules are attached with a deuterium atom. Since it is difficult to remove hydrogen $\left(\mathrm{H}_{2}\right)$ from residual gas in a vacuum, the main component of the residual gas is $\mathrm{H}_{2}$ in our sample chamber. Thus, it is considered that a certain amount of hydrogen atom $(\mathrm{H})$ is adsorbed on the Ag surface. The $\left[\left(\mathrm{D}_{2} \mathrm{O}\right)_{n}+\mathrm{D}\right]^{+}$indicate that the protons supply from inside of the cluster, not from the target surface, in the ionization reaction caused by cluster collision and decomposition. As $E / n$ increased, a shift towards smaller ions was observed in the size distribution of the dissociated components of the cluster. The dissociation rate of the cluster ions is related to the impulsive stress caused by the collision of the cluster ions with the surface $[16,17]$. 


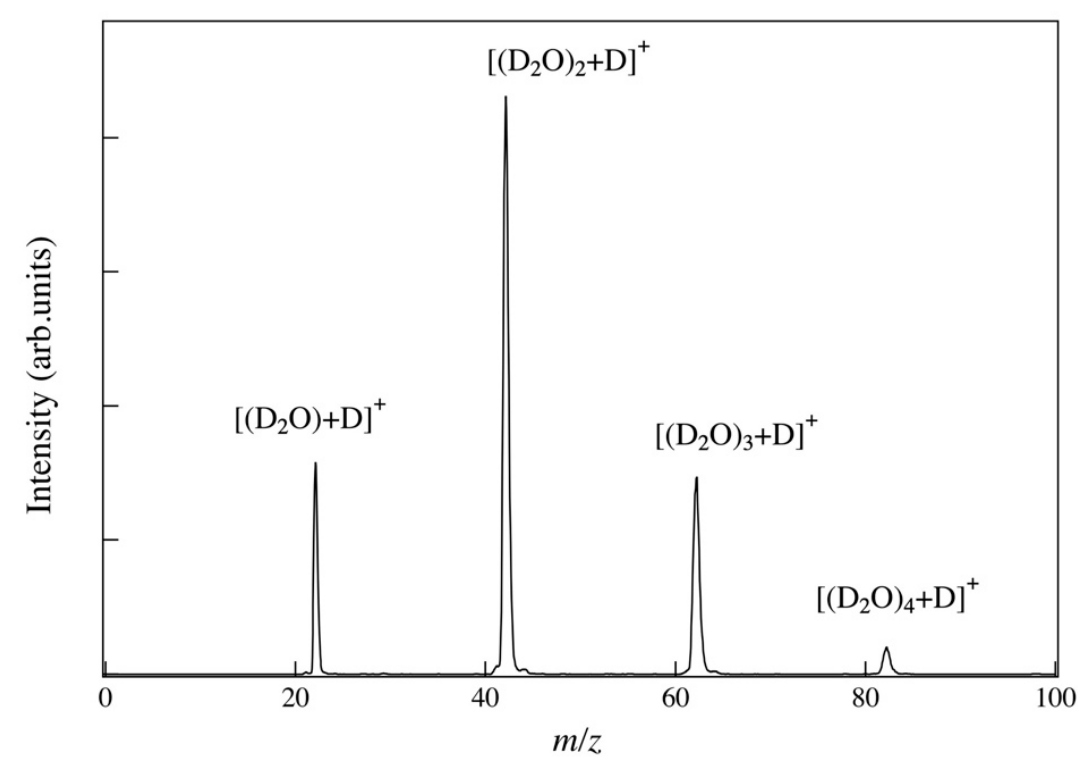

Figure 5. Mass spectra of ions detected from Ag sample under bombardment with the water cluster $\left(\mathrm{D}_{2} \mathrm{O}\right)_{900}{ }^{+}$ion at an incident angle of $45^{\circ}$.

Figure 6 shows the mass spectra of ions scattered from the Ag surface under bombardment with pure water $\left(\mathrm{H}_{2} \mathrm{O}\right)(\mathrm{a})$ and water/Ar mixed (b and c) cluster ions. The selected cluster mass is $45,000 \mathrm{Da}$, so $n=2500$ and $E / n=2.0 \mathrm{eV}$ for the pure $\mathrm{H}_{2} \mathrm{O}$ cluster. The vertical axis is the ion intensity, representing the detected ion count divided by the incident ion current. Since the E/ $n$ was lower than the case of Figure 5, the degree of dissociation of the water cluster is small. Figure $6 \mathrm{~b}, \mathrm{c}$ shows the case of a mixed cluster of water and Ar. The Ar mixing ratio is 46 and $76 \%$, respectively. The energy per projectile mass $(E / m)$ is $0.11 \mathrm{eV} / \mathrm{amu}$ for all cases of Figure $6 \mathrm{a}-\mathrm{c}$.

Here, we consider that the $\mathrm{H}_{2} \mathrm{O}$ / Ar mixed cluster beams are mainly composed of $\operatorname{Ar}_{n}\left(\mathrm{H}_{2} \mathrm{O}\right)_{n^{\prime}}$ clusters. For the mixed cluster $(b, c)$, the scattered fragments of water molecules are different from those for the $\mathrm{H}_{2} \mathrm{O}$ cluster (a). The notable difference is that as the mixing ratio of Ar increases, the proportion of the smaller water cluster $\left(\mathrm{H}_{3} \mathrm{O}^{+},\left[\left(\mathrm{H}_{2} \mathrm{O}\right)_{2}+\mathrm{H}\right]^{+}\right.$, $\left.\left[\left(\mathrm{H}_{2} \mathrm{O}\right)_{3}+\mathrm{H}\right]^{+}\right)$increases. Besides, fragments such as $\left[\mathrm{H}_{2} \mathrm{O}+\mathrm{OH}\right]^{+}$are detected in Figure $6 \mathrm{~b}, \mathrm{c}$. These fragments are produced with the breakage of $\mathrm{H}_{2} \mathrm{O}$ intramolecular bonds. In these experiments, the current density of the beams are about a few $\mathrm{nA} / \mathrm{cm}^{2}$, so the possibilities of two clusters colliding at the same site on the surface simultaneously are negligible.

The change in the dissociative scattering spectrum due to the Ar mixture ratio difference can be interpreted consistently by the change in the impact stress when the mixed clusters collide. The dissociation rate in the dissociation scattering spectrum depends on the impact stress acting on the contact region [16] The impact stress should be smaller for the water clusters than for the Ar clusters because a part of the collision energy is consumed to excite rotation and vibration inside the water molecule. For the $\mathrm{Ar}_{n}\left(\mathrm{H}_{2} \mathrm{O}\right)_{n^{\prime}}$ cluster, $\mathrm{Ar}$ atoms, which do not have the degree of freedom of vibration and rotation, attack $\mathrm{H}_{2} \mathrm{O}$ molecules at the collision time, so the dissociation of the cluster and the molecule may have been promoted.

It is difficult to exclude the possibility that the mixed cluster beam contains $\mathrm{Ar}_{\mathrm{n}}$ and $\left(\mathrm{H}_{2} \mathrm{O}\right)_{\mathrm{n}}$. The peaks of $\mathrm{Ar}_{2}{ }^{+}$and $\mathrm{Ar}_{3}{ }^{+}$are also detected in Figure $6 \mathrm{~b}, \mathrm{c}$. However, we can consider the composition of the cluster from the ratio of $\mathrm{Ar}_{2}{ }^{+} / \mathrm{Ar}_{3}{ }^{+}$. When $\mathrm{Ar}_{1500}{ }^{+}$ $(60,000 \mathrm{Da})$ is scattered on the $\mathrm{Ag}$ surface, the ratio of $\mathrm{Ar}_{2}{ }^{+}$to $\mathrm{Ar}_{3}{ }^{+}\left(\mathrm{Ar}_{2}{ }^{+} / \mathrm{Ar}_{3}{ }^{+}\right)$is about 0.47 at a collision energy of $5 \mathrm{keV}$ [16]. Since the dissociation rate increases with the incident velocity of the cluster, the values of $\mathrm{Ar}_{2}{ }^{+} / \mathrm{Ar}_{3}{ }^{+}$should be larger than 0.47 when assuming that $\mathrm{Ar}_{2}{ }^{+}$and $\mathrm{Ar}_{3}{ }^{+}$in Figure 6b,c are all generated from $\mathrm{Ar}_{1125}{ }^{+}$(45,000 Da). In Figure 6b,c, the values of $\mathrm{Ar}_{2}{ }^{+} / \mathrm{Ar}_{3}{ }^{+}$are 0.26 and 0.43 , respectively, indicating that they are mainly generated from $\mathrm{Ar}_{n}\left(\mathrm{H}_{2} \mathrm{O}\right)_{n^{\prime}}$. 


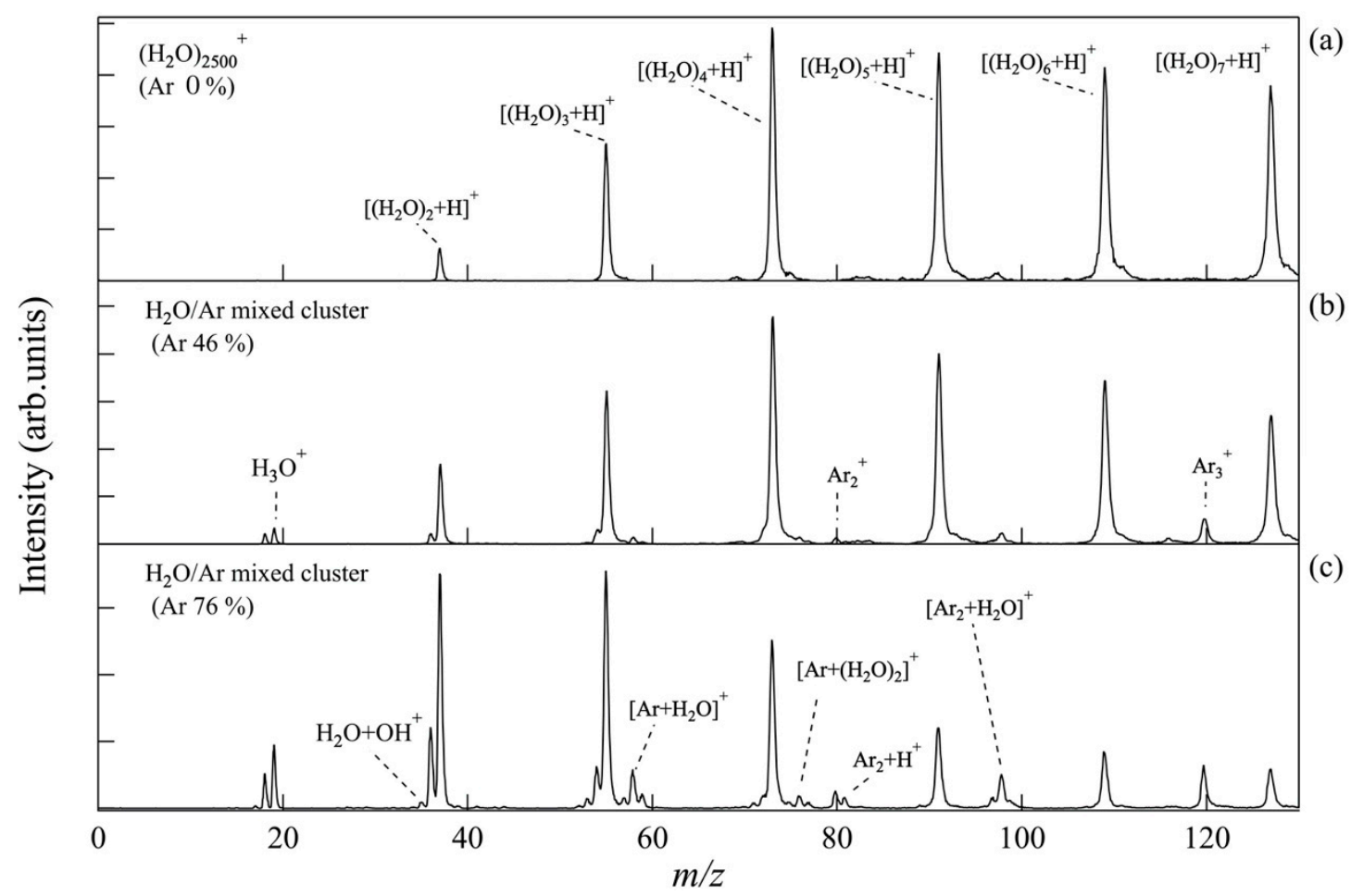

Figure 6. Mass spectra of ions detected from Ag sample under bombardment with the water cluster $\left(\mathrm{H}_{2} \mathrm{O}\right)_{2500}{ }^{+}$ion $(\mathbf{a})$ and water/Ar mixed cluster ions $(\mathbf{b}, \mathbf{c})$. The selected mass of the clusters are $45,000 \mathrm{Da}$ and $E / m$ is $0.11 \mathrm{eV} / \mathrm{amu}$.

In the mass spectrum for the mixed clusters, the peak intensities of the $\left[\left(\mathrm{H}_{2} \mathrm{O}\right)_{n}+\mathrm{H}\right]^{+}$ ions are higher than those of $\mathrm{Ar}_{\mathrm{n}}{ }^{+}$, even though Ar content of $76 \%$ in a cluster. This may be due to the difference in ionization energies of Ar and water molecules. The ionization energy of Ar cluster is $\sim 15 \mathrm{eV}$ [13], but if the water molecules were ionized by the protonation due to recombination of hydrogen bonds in a cluster, it would ionize at lower energies than Ar. Thus, the water molecules may have been preferentially ionized during the collision.

To investigate the irradiation effect of molecular cluster ions on the desorption and ionization of organic materials, we measured the SIMS spectrum using the various types of cluster ions as primary ions. We used thin films of the Asp molecule (M) as the target sample and compared the proton-attached molecular ion $[\mathrm{M}+\mathrm{H}]^{+}$intensity when $\mathrm{Ar}, \mathrm{Kr}$, water, methanol, methane, and benzene were used as the primary ion. The selected cluster size $n$ is 1500 for all beams. Figure 7 shows the relative intensities of the $[\mathrm{M}+\mathrm{H}]^{+}$ ion from Asp thin films obtained with the various primary ion beam irradiation. The vertical axis is normalized by the intensity for the Ar cluster irradiation. A remarkable enhancement of molecular ion intensity was observed for the water and the methanol cluster ion beams compared to the Ar. For these beams, peaks of $\left[\mathrm{M}+\left(\mathrm{H}_{2} \mathrm{O}\right)_{n}+\mathrm{H}\right]^{+}$and $\left[\mathrm{M}+\left(\mathrm{CH}_{3} \mathrm{OH}\right)_{n}+\mathrm{H}\right]^{+}$were detected in addition to $[\mathrm{M}+\mathrm{H}]^{+}$, respectively [15]. Such behavior of molecular ions has been reported in the previous study of water cluster ion beam $[5,6]$ and neutral $\mathrm{SO}_{2}$ cluster beam [18]. On the other hand, under the methane and benzene cluster ion bombardments, no progression of the $[\mathrm{M}+\mathrm{H}]^{+}$peaks with the $\mathrm{CH}_{4}$ or $\mathrm{C}_{6} \mathrm{H}_{6}$ attachment and also no enhancement of $[\mathrm{M}+\mathrm{H}]^{+}$were observed in the spectrum. 


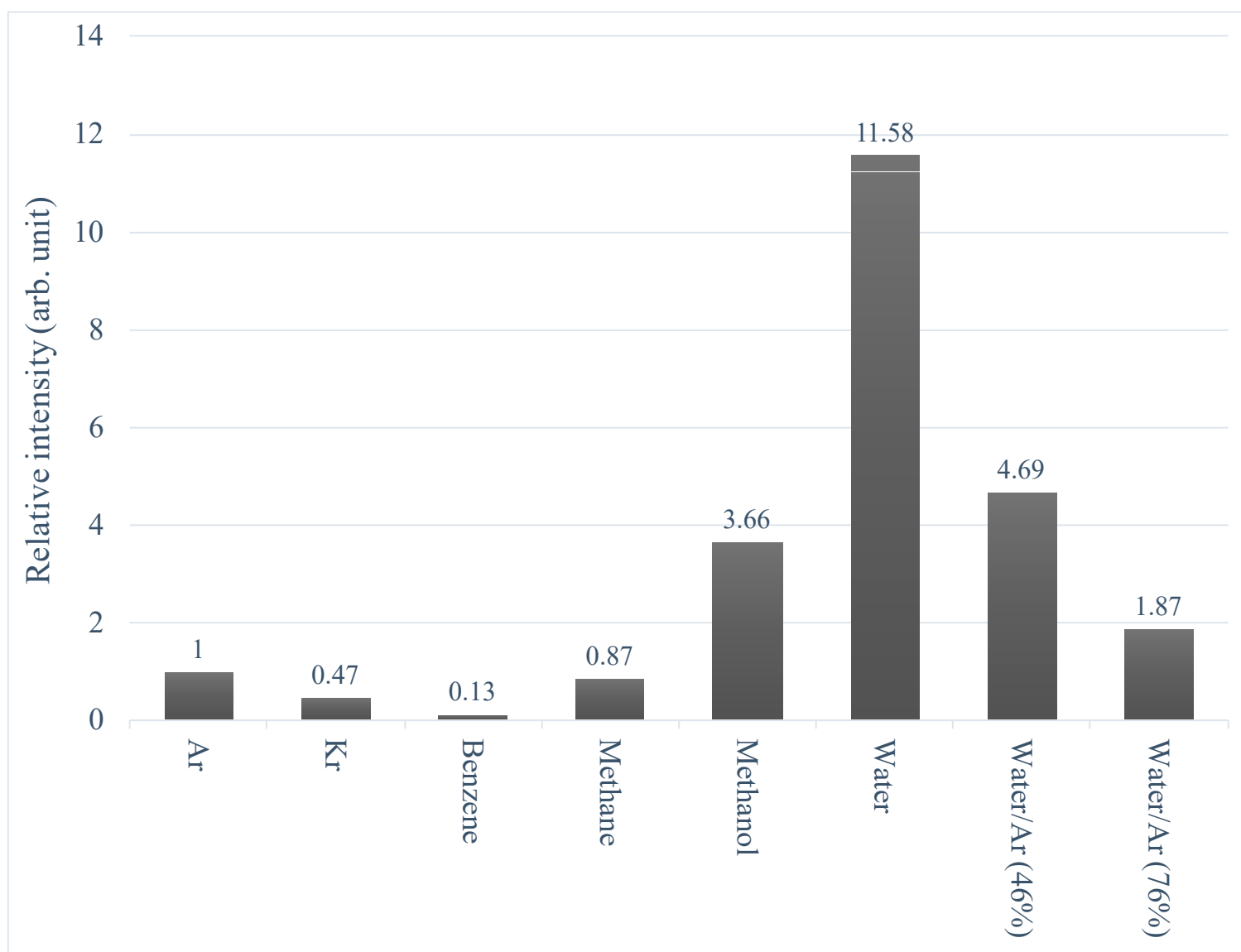

Primary ion beams

Figure 7. Relative intensities of molecular ion for aspartic acid $\left([\mathrm{M}+\mathrm{H}]^{+}, m / z=134\right)$ obtained with $\mathrm{Ar}_{1500}{ }^{+}, \mathrm{Kr}_{1500}{ }^{+}$, benzene $\left(\mathrm{C}_{6} \mathrm{H}_{6}\right)_{1500}{ }^{+}$, methane $\left(\mathrm{CH}_{4}\right)_{1500}{ }^{+}$, methanol $\left(\mathrm{CH}_{3} \mathrm{OH}\right)_{1500}{ }^{+}$, water $\left(\mathrm{H}_{2} \mathrm{O}\right)_{1500}{ }^{+}$, and water $\left(\mathrm{H}_{2} \mathrm{O}\right) /$ Ar mixed clusters primary ion beams. The acceleration voltage of the clusters is $5 \mathrm{kV}$. The selective mass of the mixed cluster is $27,000 \mathrm{Da}$, which is the same as the $\left(\mathrm{H}_{2} \mathrm{O}\right)_{1500}{ }^{+}$cluster at $m / z$. Each intensity is normalized by the intensity for the Ar cluster irradiation.

The energy per projectile mass (i.e., substantially velocity) is known to be one of the physical parameters affecting the sputtering process and thus the sputtering yields [19-21]. The molecular clusters used in this experiment have the same cluster size and total energy $(5 \mathrm{keV})$, but have different masses due to the different masses of the constituent molecules, and therefore different $E / m$. Before discussing the chemical effects caused by the molecules of the cluster, we consider the effects of cluster mass $m$. Comparing the $E / m[\mathrm{eV} / \mathrm{amu}]$ values for each projectile, $\operatorname{Ar}(0.083)$ and methanol (0.10), $\mathrm{Kr}(0.40)$ and benzene (0.43), and methane (0.21) and water (0.19) are very close, respectively. Furthermore, water and water/Ar mixed clusters have equal $E / m$ values. Comparing the relative intensities of $[\mathrm{M}+\mathrm{H}]^{+}$when using similar $E / m$ projectiles shows that $E / m$ cannot consistently explain the signal enhancement due to the molecular clusters in Figure 7. For instance, the $E / m$ of methane and water clusters are about the same, but the signal intensities are very different. (Relative intensity is 0.87 for methane but 11.6 for water.) For benzene and krypton, the $E / m$ is about the same (slightly smaller for $\mathrm{Kr}$ ), but the signal intensity is much lower for benzene (0.13) than for $\operatorname{Kr}(0.47)$. Even if $E / m$ is larger than Ar, the relative intensity increases like water and methanol or decreases like methane. Furthermore, when comparing water and water/Ar clusters, the signal intensity decreased as the Ar mixture ratio increases, even though the $E / m$ is the same. We cannot find a consistent tendency due to $E / m$ in the signal intensity between these data. The effect of $E / m$ seems to be significantly small compared to the chemical effect, at least in the energy and mass range in Figure 7. 
A possible factor to explain signal enhancement in Figure 7 is the polarity of molecules. Molecular dynamics simulations by P. Schneider [22] suggest that polar clusters increase the probability of spatter by attracting internal target molecules. This mechanism may be an essential point to unravel the signal enhancement by molecular cluster projectiles. However, since the secondary ion yield is not comparable to the sputtering yield, including neutral particles, it is unclear whether the effect can explain all the changes in secondary ion yield in Figure 7.

In terms of ionization probability, the strongly polar water and methanol molecules may promote protonation to the similarly polar molecule like Asp. The mechanism, however, does not seem to be simple. Figure 8 shows the SIMS spectra for an Asp sample measured using $\left(\mathrm{D}_{2} \mathrm{O}\right){ }_{1500}{ }^{+}$as the primary ion. The protonated molecular ion of Asp is detected, but the attached protons are mainly $\mathrm{H}$ instead of $\mathrm{D}$. Protonated ions may be formed by reaction with water dissolved in the sample [23]. However, the $\mathrm{D}_{2} \mathrm{O}$ cluster beam was irradiated below the static limit in this experiment, and we have confirmed that no $[\mathrm{M}+\mathrm{D}]+$ species are detected in the samples after $\mathrm{D}_{2} \mathrm{O}$ irradiation by SIMS measurements using Ar cluster projectiles. In addition to it, we performed SIMS measurements using Ar clusters projectile in a $\mathrm{D}_{2} \mathrm{O}$ atmosphere $\left(7.3 \times 10^{-5} \mathrm{~Pa}\right)$ and confirmed that no $[\mathrm{M}+\mathrm{D}]+$ species were detected. $[\mathrm{M}+\mathrm{D}]^{+}$species may not have been caused by $\mathrm{D}_{2} \mathrm{O}$ dissolved in the sample from the beam, but rather by the transient mixing and $\mathrm{H} / \mathrm{D}$ exchange reaction between $\mathrm{H}_{2} \mathrm{O}$ contained in the sample, the target molecule, and the $\mathrm{D}_{2} \mathrm{O}$ cluster during the cluster collision. We have also observed the enhancement of intensities of fragment ions without protons attachment [15]. In order to elucidate the mechanism of desorption/ionization of organic molecules by the molecular cluster collision, it is necessary to measure both the sputtering rate and the secondary ion yield, which is a future subject.

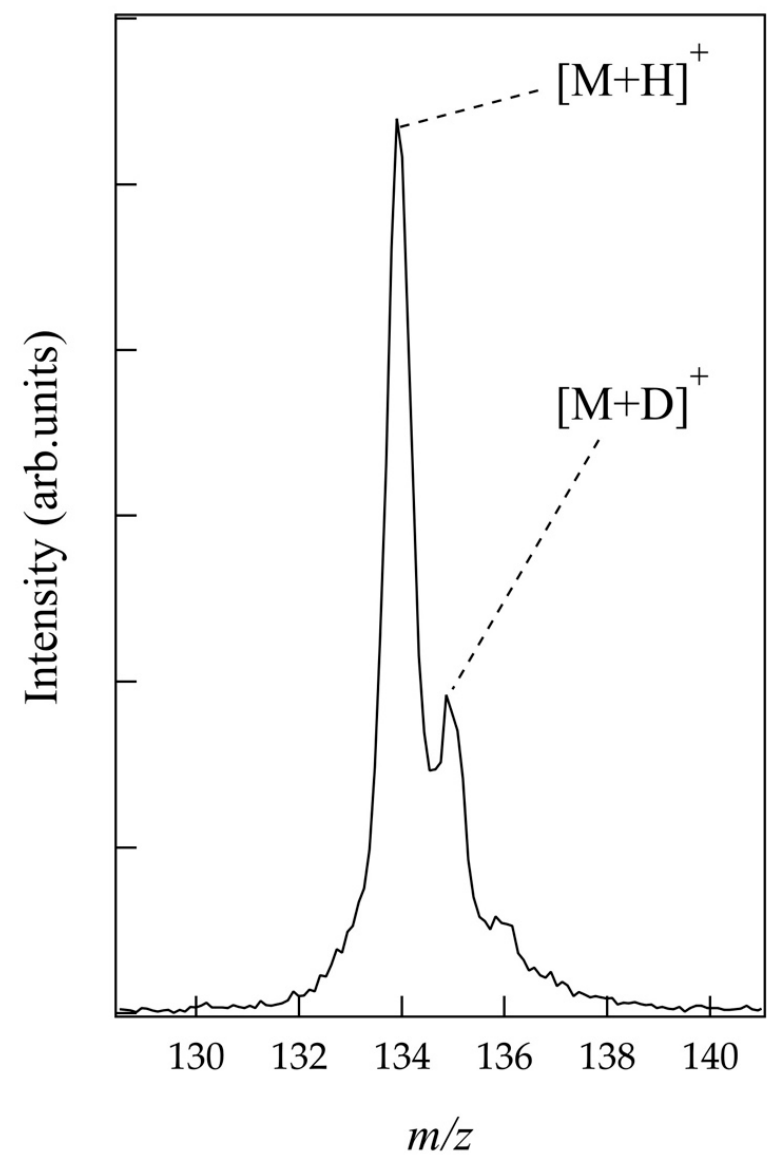

Figure 8. SIMS spectra of an Asp $\left(m / z=133\right.$ for M) film by the bombardment of $\left(\mathrm{D}_{2} \mathrm{O}\right)_{1500}{ }^{+}$at an acceleration voltage of $5 \mathrm{kV}$. The ion beam fluence is $2.0 \times 10^{11}$ ions $/ \mathrm{cm}^{2}$. 


\section{Conclusions}

We generated cluster ion beams of water, methanol, and benzene using the bubbling method and irradiated these molecular cluster ion beams to the silver and Asp thin film samples. The mass spectrum of the scattered ions and the secondary ions emitted from these samples were measured.

The mass spectrum of the scattered ions provided information on the nature of the molecular clusters and the reaction upon collision with the surface. First, the water clusters decompose when they collide onto the surface, but the molecules themselves do not dissociate even at $E / m=0.56 \mathrm{eV}(E / n=10.0 \mathrm{eV})$. However, when mixed with Ar, the water molecules are decomposed even at $E / m=0.11 \mathrm{eV}$. This may be because Ar atoms attack water molecules as the clusters collide onto the surface and collapse. The dissociated ions of the water cluster are protonated, and the protons are supplied from inside the cluster. On the other hand, the SIMS spectrum of the Asp sample shows that the protons are mainly supplied to Asp from outside the cluster. Experiments using $\left(\mathrm{D}_{2} \mathrm{O}\right)_{n}{ }^{+}$may provide a key to elucidating the dynamics of organic molecular protonation by molecular cluster irradiation.

From a result of the SIMS measurement of Asp molecules, remarkable enhancement of $[\mathrm{M}+\mathrm{H}]^{+}$ion intensity was observed in the water and the methanol clusters. On the other hand, no enhancement was observed for methane and benzene clusters. At this time, the detailed mechanism of secondary ion formation is still unknown, but systematic studies using various types of molecular cluster ions with different chemical properties may reveal the basic mechanism of secondary ion formation.

Author Contributions: K.M. wrote the manuscript. K.M., S.N., A.T. and K.G. performed the experiments and the data analysis. K.M. supervised the research. N.I. reviewed and edited the manuscript. All authors have read and agreed to the published version of the manuscript.

Funding: This work was supported by JSPS KAKENHI Grant Number JP16K05821.

Data Availability Statement: The data presented in this study are available on request from the corresponding author.

Acknowledgments: The authors would like to thank Masanori Kanai for the assistance with the experiments. The authors also appreciate Noriaki Toyoda for his help in measuring film thickness of the target samples.

Conflicts of Interest: The authors declare no conflict of interest.

\section{Appendix A}

We measured the change in the background pressure of water in the irradiation chamber during beam irradiation using Ar and He as the carrier gas, respectively. As a result, the amount of water entering the irradiation chamber was almost the same at a carrier gas flow rate of $260 \mathrm{sccm}$, suggesting that the He carrier gas also takes in enough water. Besides, the size distribution of water clusters was measured when the carrier gas was a mixture of Ar and He. Figure A1 shows the size distribution of water clusters generated using Ar and Ar/He carrier gases. The size distribution of the water cluster shifts to the smaller side by mixing He with the Ar carrier gas.

Furthermore, when the Ar/He mixing ratio was varied, the cluster size became smaller as the He mixing ratio increased. These results indicate that He is inhibiting the clustering of water molecules. He, which has a mass close to that of hydrogen, may excite the vibrations of hydrogen atoms by collisions, and, as a result, the internal energy of water molecules is not sufficiently reduced. 


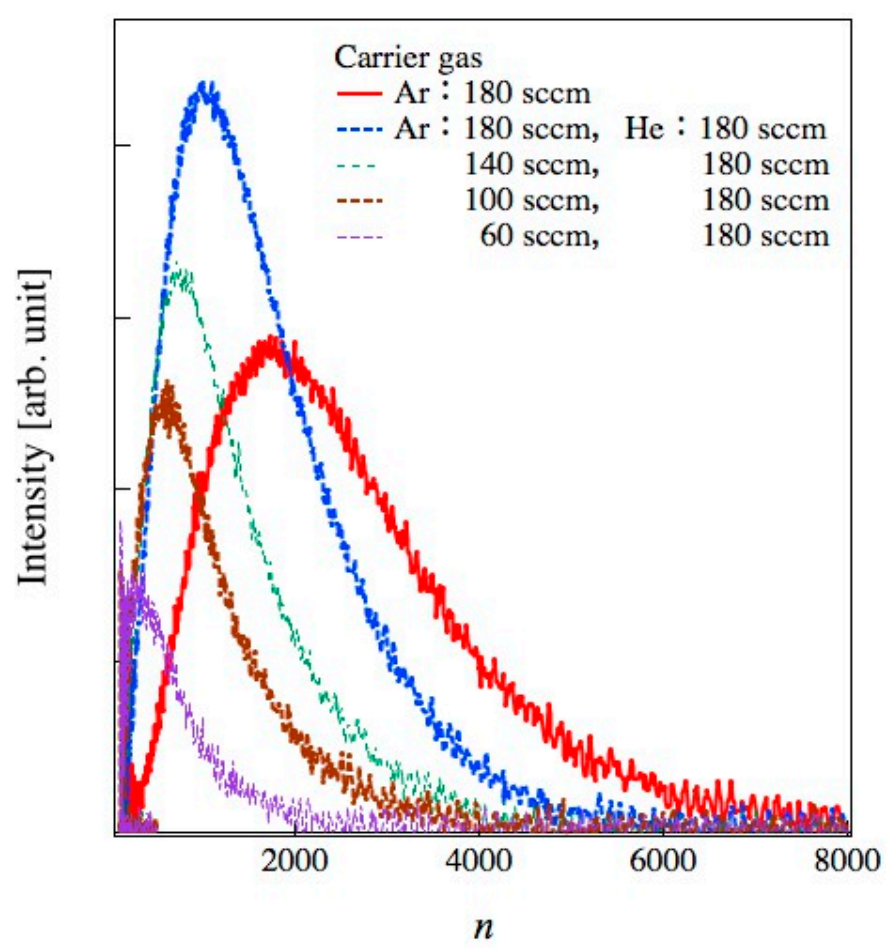

Figure A1. Size ( $n$ ) distribution of water cluster ion beams generated by Ar and Ar/He carrier gases.

\section{References}

1. Toyoda, N.; Matsuo, J.; Aoki, T.; Yamada, I.; Fenner, B.D. Secondary ion mass spectrometry with gas cluster ion beams. Nucl. Instr. Meth. B 2002, 190, 860-864. [CrossRef]

2. Ninomiya, S.; Ichiki, K.; Yamada, H.; Nakata, Y.; Seki, T.; Aoki, T.; Matsuo, J. Precise and fast secondary ion mass spectrometry depth profiling of polymer materials with large Ar cluster ion beams. Rapid Commun. Mass Spectrom. 2009, 23, $1601-1606$. [CrossRef] [PubMed]

3. Mochiji, K.; Hashinokuchi, M.; Moritani, K.; Toyoda, N. Matrix-free detection of intact ions from proteins in argon-cluster secondary ion mass spectrometry. Rapid Commun. Mass Spectrom. 2009, 23, 648-652. [CrossRef] [PubMed]

4. Moritani, K.; Kanai, M.; Goto, K.; Ihara, I.; Inui, N.; Mochiji, K. Secondary ion emission from insulin film bombarded with methane and noble gas cluster ion beams. Nucl. Instr. Meth. Phys. Res. B 2013, 415, 300-303. [CrossRef]

5. Sheraz, S.; Barber, A.; Fletcher, J.S.; Locker, N.P.; Vickerman, J.C. Enhancing Secondary Ion Yields in Time of Flight-Secondary Ion Mass Spectrometry Using Water Cluster Primary Beams. Anal. Chem. 2013, 85, 5654-5658. [CrossRef]

6. Rabbani, S.; Razo, I.B.; Kohn, T.; Lockyer, N.P.; Vickerman, J.C. Enhancing Ion Yields in Time-of-Flight-Secondary Ion Mass Spectrometry: A Comparative Study of Argon and Water Cluster Primary Beams. Anal. Chem. 2015, 87, 2367-2374. [CrossRef]

7. Sheraz, S.; Tian, H.; Vickerman, J.C.; Blenkinsopp, P.; Winograd, N.; Cumpson, P. Enhanced Ion Yields Using High Energy Water Cluster Beams for Secondary Ion Mass Spectrometry Analysis and Imaging. Anal. Chem. 2019, 91, 9058-9068. [CrossRef]

8. Wucher, A.; Tian, H.; Winograd, N. A mixed cluster ion beam to enhance the ionization efficiency in molecular secondary ion mass spectrometry. Rapid Commun. Mass Spectrom. 2014, 28, 396-400. [CrossRef]

9. Tian, H.; Wucher, A.; Winograd, N. Dynamic Reactive Ionization with Cluster Secondary Ion Mass Spectrometry. J. Am. Soc. Mass Spectrom. 2016, 27, 285-292. [CrossRef]

10. Moritani, K.; Hashinokuchi, M.; Mukai, G.; Mochiji, K. New Design and Development of Size-Selected Gas Cluster SIMS. Electr. Eng. Jpn. 2011, 176, 52-58. [CrossRef]

11. Hagena, O.F. Condensation in free jets: Comparison of rare gases and metals. Z. Phys. D 1987, 4, 291-299. [CrossRef]

12. Smith, R.A.; Ditmire, T.; Tisch, J.E.G. Characterization of a cryogenically cooled high-pressure gas jet for laser/cluster interaction experiments. Rev. Sci. Instrum. 1998, 69, 3798-3804. [CrossRef]

13. Pauly, H. The Role of Molecular Beams in the 20th Century. In Atom, Molecule, and Cluster Beams; Springer: Berlin/Heidelberg, Germany, 2000; Volume 28, pp. 1-34.

14. Koišek, J.; Lengyel, J.; Fárník, M.; Slavíek, P. Energy and charge transfer in ionized argon coated water clusters. J. Chem. Phys. 2013, 139, 214308. [CrossRef]

15. Moritani, K. Enhancement of Intact Ion Yields in ToF-SIMS Using Molecular Cluster Ion Beams. Vac. Surf. Sci. 2018, 61, 452-457. (In Japanese) [CrossRef]

16. Mochiji, K.; Se, N.; Inui, N.; Moritani, K. Mass spectrometric analysis of the dissociation of argon cluster ions in collision with several kinds of metal. Rapid Commun. Mass Spectrom. 2014, 28, 2141-2146. [CrossRef] 
17. Mochiji, K.; Inui, N.; Asa, R.; Moritani, K. Argon Cluster Ions Cleaning and Probing a Graphene Layer on Copper. e-J. Surf. Sci. Nanotech 2015, 13, 167-173. [CrossRef]

18. Gebhardt, C.R.; Tomsic, A.; Schröder, H.; Dürr, M.; Kompa, K.L. Matrix-Free Formation of Gas-Phase Biomolecular Ions by Soft Cluster-Induced Desorption. Angew. Chem. Int. Ed. 2009, 48, 4162-4165. [CrossRef]

19. Delcorte, A.; Garrison, B.J.; Hamraoui, K. Dynamics of Molecular Impacts on Soft Materials: From Fullerenes to Organic Nanodrops. Anal. Chem. 2009, 81, 6676-6686. [CrossRef]

20. Delcorte, A.; Restrepo, O.A.; Czerwinski, B.; Garrison, B.J. Surface sputtering with nanoclusters: The relevant parameters. Surf. Interface Anal. 2012, 45, 9-13. [CrossRef]

21. Delcorte, A.; Moshkunov, K.; Debongnie, M. Relationships between crater and sputtered material characteristics in large gas cluster sputtering of polymers: Results from molecular dynamics simulations. J. Vac. Sci. Technol. B 2018, 36, 03F109. [CrossRef]

22. Schneider, P.; Dürr, M. Cluster-induced desorption investigated by means of molecular dynamics simulations-Microsolvation in clusters of polar and non-polar constituents. J. Chem. Phys. 2019, 150, 214301. [CrossRef]

23. Portz, A.; Gebhardt, C.R.; Dürr, M. Real-Time Investigation of the H/D Exchange Kinetics of Porphyrins and Oligopeptides by Means of Neutral Cluster-Induced Desorption/Ionization Mass Spectrometry. J. Phys. Chem. B 2017, 121, 11031. [CrossRef] 